\title{
NEW FARKAS-TYPE CONSTRAINT QUALIFICATIONS IN CONVEX INFINITE PROGRAMMING*
}

\author{
Nguyen Dinh ${ }^{1}$, Miguel A. Goberna ${ }^{2}$, Marco A. López ${ }^{2}$ and Ta Quang Son ${ }^{3}$
}

\begin{abstract}
This paper provides KKT and saddle point optimality conditions, duality theorems and stability theorems for consistent convex optimization problems posed in locally convex topological vector spaces. The feasible sets of these optimization problems are formed by those elements of a given closed convex set which satisfy a (possibly infinite) convex system. Moreover, all the involved functions are assumed to be convex, lower semicontinuous and proper (but not necessarily real-valued). The key result in the paper is the characterization of those reverse-convex inequalities which are consequence of the constraints system. As a byproduct of this new versions of Farkas' lemma we also characterize the containment of convex sets in reverse-convex sets. The main results in the paper are obtained under a suitable Farkas-type constraint qualifications and/or a certain closedness assumption.
\end{abstract}

Mathematics Subject Classification. 90C25, 90C34, 90C46, 90C48.

Received January 2, 2006.

Published online June 20, 2007.

\section{InTRODUCTION}

This paper deals with optimization problems of the form

$$
\begin{aligned}
\text { Minimize } & f(x) \\
\text { subject to } & f_{t}(x) \leq 0, t \in T, \\
& x \in C,
\end{aligned}
$$

where $T$ is an arbitrary (possibly infinite) index set, $C$ is a non-empty closed convex subset of a locally convex Hausdorff topological vector space $X$, and $f, f_{t}: X \rightarrow \mathbb{R} \cup\{+\infty\}, t \in T$, are proper lower semicontinuous (l.s.c., in brief) convex functions.

\footnotetext{
Keywords and phrases. Convex infinite programming, KKT and saddle point optimality conditions, duality theory, Farkas-type constraint qualification.

* This research was partially supported by MEC of Spain and FEDER of EU, Grant MTM2005-08572-C03-01, and by Project B.2005.23.68 of the MOET, Vietnam.

1 Department of Mathematics, International University, Vietnam National University-HCM city, Linh Trung ward, Thu Duc district, Ho Chi Minh city, Vietnam.

2 Department of Statistics and Operations Research, University of Alicante, 03080 Alicante, Spain; Marco.Antonio@ua.es

3 Nha Trang College of Education, Nha Trang, Vietnam.
} 
Throughout the paper we assume that the (convex) constraint system

$$
\sigma:=\left\{f_{t}(x) \leq 0, t \in T ; x \in C\right\}
$$

is consistent, with solution set represented by $A(A \neq \emptyset)$.

The system $\sigma$ is called linear when $f_{t}(x)=a_{t}(x)-b_{t}, a_{t} \in X^{*}$ (topological dual of $X$ ), $b_{t} \in \mathbb{R}, t \in T$, and $C=X$. Moreover, it is called infinite (ordinary or finite) if the dimension of $X$ and the number of constraints $(|T|)$ are infinite (finite, respectively). If exactly one of these numbers is finite, then $\sigma$ is called semi-infinite (typically, $T$ is infinite and $X=\mathbb{R}^{n}$ ). An optimization problem is called infinite (finite, semi-infinite) when its constraint system is infinite (finite, semi-infinite, respectively).

The objective of the paper is to provide optimality conditions, duality theorems, and stability theorems for $(\mathrm{P})$. To do that we introduce new Farkas-type constraint qualifications and new versions of Farkas lemma. The classical Farkas lemma characterizes those linear inequalities which are consequences of a consistent ordinary linear inequality system (i.e., they are satisfied by every solution of the system). Farkas-type results for convex systems (characterizing families of inequalities which are consequences of a consistent convex system $\sigma$ ) are fundamental in convex optimization and in other fields as game theory, set containment problems, etc. Since the literature on Farkas lemma, and its extensions, is very wide (see, e.g., the survey in [15]), we just mention here some works giving Farkas-type results for the kind of systems considered in the paper: [3, 11, 16, 22] for semi-infinite systems, $[9,14,17,21]$ for infinite systems, and $[8,18,19]$ for cone convex systems.

The paper is organized as follows. Section 2 contains the necessary notations and recalls some basic results on convexity and convex systems. Section 3 extends to infinite convex systems two constraint qualifications (c.q., in brief) which play a crucial role in linear semi-infinite programming, one of them (the so-called FarkasMinkowski property, FM in brief) being of global nature whereas the other one is a local property (and so it is called locally Farkas-Minkowski, LFM in short). Section 4 provides new asymptotic and non-asymptotic versions of Farkas' lemma characterizing those reverse-convex inequalities $f(x) \geq \alpha$ which are consequences of $\sigma$. The non-asymptotic Farkas' lemma requires the FM c.q. together with a certain closedness condition involving $f_{t}, t \in T$, and $f$ (which holds whenever $f$ is linear or it is continuous at some feasible point), and it provides a characterization of the containment of convex sets in reverse-convex sets. Under these two assumptions we obtain, in Section 5, a Karush-Kuhn-Tucker (KKT) optimality condition for (P), we show that the LFM c.q. holds everywhere if the constraint system is FM, and, what is more important, that the LFM c.q. is, in a certain sense, the weakest condition guarateeing that $(\mathrm{P})$ satisfies the KKT condition at the optimal solutions. Finally, in Section 6, a strong duality theorem and an optimality condition for $(\mathrm{P})$, in terms of saddle points of the associated Lagrange function, are established. The strong duality theorem allows us to show that the optimal value of $(\mathrm{P})$ is stable (in different senses) relatively to small arbitrary perturbations of the right-hand side function (the null function).

\section{Preliminaries}

For a set $D \subset X$, the closure of $D$ will be denoted by $\operatorname{cl} D$ and the convex cone generated by $D \cup\{0\}$ by cone $D$. The closure with respect to the weak*-topology of a subset $E$ of either the dual space $X^{*}$ or the product space $X^{*} \times \mathbb{R}$ will be represented also by $\mathrm{cl} E$.

We represent by $\mathbb{R}_{+}^{(T)}$ the positive cone in $\mathbb{R}^{(T)}$, the so-called space of generalized finite sequences $\lambda=\left(\lambda_{t}\right)_{t \in T}$ such that $\lambda_{t} \in \mathbb{R}$, for each $t \in T$, and with only finitely many $\lambda_{t}$ different from zero. The supporting set of $\lambda \in \mathbb{R}^{(T)}$ is $\operatorname{supp} \lambda:=\left\{t \in T \mid \lambda_{t} \neq 0\right\}$. Observe that $\mathbb{R}^{(T)}$ is the topological dual of $\mathbb{R}^{T}$, endowed with the product topology, and

$$
\lambda(u)=\sum_{t \in T} \lambda_{t} u_{t}:=\sum_{t \in \operatorname{supp} \lambda} \lambda_{t} u_{t}, \forall u \in \mathbb{R}^{T}, \forall \lambda \in \mathbb{R}^{(T)}
$$


Given $\lambda \in \mathbb{R}_{+}^{(T)}$, we define

$$
\sum_{t \in T} \lambda_{t} f_{t}:=\sum_{t \in \operatorname{Supp} \lambda} \lambda_{t} f_{t}
$$

Analogously, if $\left\{Y_{t}, t \in \operatorname{supp} \lambda\right\}$ is a class of non-empty subsets of some linear space, we define also

$$
\sum_{t \in T} \lambda_{t} Y_{t}:=\sum_{t \in \operatorname{Supp} \lambda} \lambda_{t} Y_{t}
$$

so that cone $D=\left\{\sum_{t \in T} \lambda_{t} D \mid \lambda \in \mathbb{R}_{+}^{(T)}\right\}$. Let further $I$ be an arbitrary index set, $\left\{Y_{i}, i \in I\right\}$ be a family of subsets of some linear space, and let $\Im$ be the collection of all the non-empty finite subsets of $I$. Then

$$
\text { cone } \begin{aligned}
\left(\bigcup_{i \in I} Y_{i}\right) & =\bigcup_{J \in \Im} \operatorname{cone}\left(\bigcup_{j \in J} Y_{j}\right) \\
& =\bigcup_{J \in \Im}\left(\sum_{j \in J} \operatorname{cone} Y_{j}\right) .
\end{aligned}
$$

For a set $D \subset X$, the indicator function $\delta_{D}$ is defined as $\delta_{D}(x)=0$ if $x \in D$, and $\delta_{D}(x)=+\infty$ if $x \notin D$. If $D$ is a non-empty closed convex set, then $\delta_{D}$ is a proper l.s.c. convex function. The normal cone of $D$ at $x$ is given by

$$
N_{D}(x):=\left\{u \in X^{*} \mid u(y-x) \leq 0 \text { for all } y \in D\right\}
$$

if $x \in D$, and $N_{D}(x)=\varnothing$, otherwise.

Now let $h: X \rightarrow \mathbb{R} \cup\{+\infty\}$ be a proper l.s.c. convex function. The effective domain, the graph, and the epigraph of $h$ are

$$
\begin{gathered}
\operatorname{dom} h=\{x \in X \mid h(x)<+\infty\}, \\
\operatorname{gph} h=\{(x, h(x)) \in X \times \mathbb{R} \mid x \in \operatorname{dom} h\},
\end{gathered}
$$

and

$$
\text { epi } h=\{(x, \gamma) \in X \times \mathbb{R} \mid x \in \operatorname{dom} h, h(x) \leq \gamma\},
$$

respectively, whereas the conjugate function of $h, h^{*}: X^{*} \rightarrow \mathbb{R} \cup\{+\infty\}$, is defined by

$$
h^{*}(v):=\sup \{v(x)-h(x) \mid x \in \operatorname{dom} h\} .
$$

It is well-known that $h^{*}$ is also a proper l.s.c. convex function, and that its conjugate, denoted by $h^{* *}$, coincides with $h$.

The support function of $D \subset X$ is

$$
\sup _{x \in D} u(x)=\delta_{D}^{*}(u)=\delta_{\mathrm{cl}(\operatorname{conv} D)}^{*}(u), u \in X^{*} .
$$

Lemma 1. Let $g, h: X \rightarrow \mathbb{R} \cup\{+\infty\}$ be proper l.s.c. convex functions such that at least one of them is continuous at some point of $\operatorname{dom} g \cap \operatorname{dom} h$. Then epi $g^{*}+\operatorname{epi} h^{*}$ is weak $k^{*}$-closed.

Proof. If, for instance, $g$ is continuous at $c \in \operatorname{dom} h$, it is clear that $c \in \operatorname{int}(\operatorname{dom} g) \cap \operatorname{dom} h$, and this implies that 0 belongs to the core of $\operatorname{dom} g-\operatorname{dom} h$, which, in turn, entails that cone $(\operatorname{dom} g-\operatorname{dom} h)$ is a closed space. Then, it follows from [4], Proposition 3.1, that the set epi $g^{*}+\mathrm{epi}^{*} h^{*}$ is weak ${ }^{*}$-closed.

We also define the subdifferential of $h$ at $a \in \operatorname{dom} h$ as

$$
\partial h(a):=\left\{u \in X^{*} \mid h(x) \geq h(a)+u(x-a) \forall x \in X\right\} .
$$

Thus, if $D$ is a non-empty closed convex set, then $\partial \delta_{D}(a)=N_{D}(a)$ for all $a \in D$. 
On the other hand, for $g$ and $h$ as in Lemma 1, we have $\partial g(a)+\partial h(a) \subset \partial(g+h)(a)$ for all $a \in \operatorname{dom} g \cap \operatorname{dom} h$, where the inclusion can be strict. The following lemma was established in [4], Theorem 3.1, assuming that $X$ is a Banach space, but the proof is exactly the same for locally convex vector spaces.

If $g, h: X \rightarrow \mathbb{R} \cup\{+\infty\}$ are proper l.s.c. convex functions, then it is known that

$$
\operatorname{epi}(g+h)^{*}=\operatorname{cl}\left\{\operatorname{epi} g^{*}+\operatorname{epi} h^{*}\right\} .
$$

Lemma 2. Let $g, h: X \rightarrow \mathbb{R} \cup\{+\infty\}$ be proper l.s.c. convex functions. If epi $g^{*}+$ epi $^{*}$ is weak $k^{*}$-closed then, for each $a \in \operatorname{dom} g \cap \operatorname{dom} h$,

$$
\partial(g+h)(a)=\partial g(a)+\partial h(a) .
$$

For $\varepsilon>0$, the $\varepsilon$-subdifferential of $h$ at $a \in \operatorname{dom} h$ is defined as the non-empty weak*-closed convex set

$$
\partial_{\varepsilon} h(a):=\left\{v \in X^{*} \mid h(x) \geq h(a)+v(x-a)-\varepsilon, \forall x \in X\right\} .
$$

It is worth observing that, if $a \in \operatorname{dom} h$, then (proved in [14], Lem. 2.1, in Banach spaces)

$$
\operatorname{epi}^{*}=\bigcup_{\varepsilon \geq 0}\left\{\left(v, v(a)+\varepsilon-h(a) \mid v \in \partial_{\varepsilon} h(a)\right\} .\right.
$$

The characteristic cone of $\sigma=\left\{f_{t}(x) \leq 0, t \in T ; x \in C\right\}$ is

$$
K:=\text { cone }\left\{\bigcup_{t \in T} \operatorname{epi} f_{t}^{*} \cup \operatorname{epi} \delta_{C}^{*}\right\} .
$$

Taking into account that epi $\delta_{C}^{*}$ is a convex cone, we can also write

$$
K:=\operatorname{cone}\left\{\bigcup_{t \in T} \operatorname{epi} f_{t}^{*}\right\}+\operatorname{epi} \delta_{C}^{*} .
$$

Since $A \neq \emptyset$, and given $v \in X^{*}$ and $\alpha \in \mathbb{R}$,

$$
v(x) \leq \alpha \text { is a consequence of } \sigma \Leftrightarrow(v, \alpha) \in \operatorname{cl} K .
$$

([9], Th. 4.1, extending [16], Th. 3.2.)

\section{FARKAS-TYPE CONSTRAINT QUALIFICATIONS}

The following constraint qualification was introduced in [5] as a very general assumption for the duality theorem in linear semi-infinite programming, and it has also been used in convex programming (see, e.g., [8]).

Definition 1. We say that $\sigma$ is Farkas-Minkowski (FM, in brief) if $K$ is weak*-closed.

If cone $\left\{\bigcup_{t \in T} \operatorname{gph} f_{t}^{*} \cup \operatorname{gph} \delta_{C}^{*}\right\}$ is weak*-closed, then $\sigma$ is FM [9], Prop. 3.4). The converse is not true.

Observe that $\left\{\delta_{D}(x) \leq 0\right\}$ is a FM representation of any closed convex set $D \neq \emptyset$, because epi $\delta_{D}^{*}$ is a weak*closed cone. In particular, $\left\{\delta_{A}(x) \leq 0\right\}$ is a FM system which has the same solutions as $\sigma$ and, so, the same continuous linear consequences (inequalities of the form $v(x) \leq \alpha$, with $v \in X^{*}$ and $\alpha \in \mathbb{R}$ ); i.e.,

$$
\operatorname{cl} K=\operatorname{epi} \delta_{A}^{*}
$$

(This statement extends [16], (4.2).)

If $S \subset T$ and $|S|<\infty$, then $\sigma_{S}:=\left\{f_{t}(x) \leq 0, t \in S ; x \in C\right\}$ is a finite subsystem of $\sigma$. 
Proposition 1. If $\sigma$ is FM, then every continuous linear consequence of $\sigma$ is also consequence of a finite subsystem of $\sigma$. The converse statement holds if $\sigma$ is linear.

Proof. Let $\sigma$ be FM. If $v(x) \leq \alpha$, with $v \in X^{*}$, is consequence of $\sigma$, then $(v, \alpha) \in \operatorname{cl} K=K$ and, by (2.1), there exist $S \subset T$, with $|S|<\infty,\left\{u_{t}, t \in S ; w\right\} \subset X^{*}$, and $\left\{\lambda_{t}, t \in S ; \alpha_{t}, t \in S ; \mu ; \beta\right\} \subset \mathbb{R}_{+}$such that

$$
(v, \alpha)=\sum_{t \in S} \lambda_{t}\left(u_{t}, f^{*}\left(u_{t}\right)+\alpha_{t}\right)+\mu\left(w, \delta_{C}^{*}(w)+\beta\right) \in K_{S},
$$

where $K_{S}$ denotes the characteristic cone of $\sigma_{S}$. Since $(v, \alpha) \in \operatorname{cl} K_{S}, v(x) \leq \alpha$ is consequence of $\sigma_{S}$.

Now let $C=X$ and $f_{t}(x)=a_{t}(x)-b_{t}$, with $a_{t} \in X^{*}$ and $b_{t} \in \mathbb{R}, t \in T$. Since $f_{t}^{*}=b_{t}+\delta_{\left\{a_{t}\right\}}, t \in T$, and $\delta_{X}^{*}=\delta_{\{0\}}$, we have $K=$ cone $\left\{\left(a_{t}, b_{t}\right), t \in T ;(0,1)\right\}$.

Let $(v, \alpha) \in \operatorname{cl} K$. This is equivalent to assert that $v(x) \leq \alpha$ is consequence of $\sigma$. By assumption, there exists $S \subset T$, with $|S|<\infty$, such that $v(x) \leq \alpha$ is consequence of $\sigma_{S}$, so that $(v, \alpha) \in \operatorname{cl} K_{S}$, where $K_{S}$ denotes again the characteristic cone of $\sigma_{S}$; i.e., $K_{S}=$ cone $\left\{\left(a_{t}, b_{t}\right), t \in S ;(0,1)\right\}$. Since this cone is finite dimensional, it is weak ${ }^{*}$-closed and $(v, \alpha) \in K_{S} \subset K$. Thus, $K$ is weak ${ }^{*}$-closed.

The following example shows that the converse statement of Proposition 1 is not true for convex systems (even though $X=C=\mathbb{R}^{n}$ and $|T|=1$ ).

Example 1. Let $X=C=\mathbb{R}^{n}, T=\{1\}$, and $\sigma=\left\{f_{1}(x):=\frac{1}{2}\|x\|^{2} \leq 0\right\}$. Since $f_{1}^{*}(v)=\frac{1}{2}\|v\|^{2}, K=$ $\left(\mathbb{R}^{n} \times \mathbb{R}_{++}\right) \cup\{0\}$ is not closed. Thus, $\sigma$ is a finite non-FM convex system.

The following version of Farkas lemma ([9], Th. 4.4) will be used later on.

Lemma 3. Let $\sigma$ be FM, $v \in X^{*}$, and $\alpha \in \mathbb{R}$. Then, the following statements are equivalent:

(i) $v(x) \geq \alpha$ is consequence of $\sigma$;

(ii) $(-v,-\alpha) \in K$;

(iii) there exists $\lambda \in \mathbb{R}_{+}^{(T)}$ such that

$$
v(x)+\sum_{t \in T} \lambda_{t} f_{t}(x) \geq \alpha, \forall x \in C
$$

Let us introduce another constraint qualification. Given $x \in X$, consider the indices subset

$$
T(x):=\left\{t \in T \mid f_{t}(x)=0\right\} .
$$

If $z \in A, T(z)$ is the set of indices corresponding to the active constraints at $z$, and it can be verified easily that

$$
N_{C}(z)+\text { cone }\left(\bigcup_{t \in T(z)} \partial f_{t}(z)\right) \subseteq N_{A}(z) .
$$

Definition 2. We say that $\sigma$ in (1.1) is locally Farkas-Minkowski (LFM, in short) at $z \in A$ if

$$
N_{A}(z) \subseteq N_{C}(z)+\text { cone }\left(\bigcup_{t \in T(z)} \partial f_{t}(z)\right) .
$$

$\sigma$ is said to be LFM if it is LFM at every feasible point $z \in A$. 
Thanks to (3.2), $\sigma$ is LFM at $z \in A$ if and only if

$$
N_{A}(z)=N_{C}(z)+\text { cone }\left(\bigcup_{t \in T(z)} \partial f_{t}(z)\right)
$$

The LFM property, under the name of basic constraint qualification (BCQ), was introduced in [13], p. 307, in relation to an ordinary convex programming problem, with equality/inequality constraints. It was extended in [24] to the framework of linear semi-infinite systems in the Euclidean space, and deeply studied in [11], Chapter 5. The consequences of its extension to convex semi-infinite systems were analyzed in [10].

In [22] and [21], the following indices subset is considered, instead of $T(x)$,

$$
\widetilde{T}(x):=\left\{t \in T \mid \varphi(x)=f_{t}(x)\right\}
$$

where $\varphi$ is the supremum function

$$
\varphi(x):=\sup _{t \in T} f_{t}(x) .
$$

Since $A \neq \emptyset, \varphi$ is a proper l.s.c. convex function (epi $\varphi=\bigcap_{t \in T} \operatorname{epi} f_{t}$ ). In [22] and [21] the continuity of $\varphi$ on $X$ is assumed, and they formulate the so-called $B C Q$ condition at $z$ as follows:

$$
(\mathrm{BCQ}): \quad N_{A}(z) \subseteq N_{C}(z)+\text { cone }\left(\bigcup_{t \in \widetilde{T}(z)} \partial f_{t}(z)\right)
$$

Whereas $X$ is the Euclidean space in [22], and it is a Banach space in [21], our LFM condition is given in a locally convex Hausdorff topological vector space $X$ and the strong requirement of the continuity of $\varphi$ on $X$ is removed. Nevertheless, the relationship between both conditions is shown in the following result.

Proposition 2. If $\varphi$ is continuous at $z \in A$ and $z$ is an interior point of $C$, the conditions LFM and BCQ at $z$, as they are respectively formulated in (3.3) and (3.4), are equivalent.

Proof. $T(z) \subset \widetilde{T}(z)$ because

$$
t \in T(z) \Rightarrow 0=f_{t}(z) \leq \varphi(z) \leq 0 \Rightarrow t \in \widetilde{T}(z) .
$$

If $\varphi(z)<0$, the continuity assumption entails that $z$ is an interior point of $A$. Then, $N_{A}(z)=\{0\}$ and $(3.3)$ and (3.4) are both trivially satisfied.

If $\varphi(z)=0$, then $T(z)=\widetilde{T}(z)$ because

$$
t \in \widetilde{T}(z) \Rightarrow f_{t}(z)=\varphi(z)=0 \Rightarrow t \in T(z) .
$$

Once again (3.3) and (3.4) are equivalent.

The following proposition is a LFM counterpart of Proposition 1.

Proposition 3. Let $z \in A$. If $\sigma$ is LFM at $z$ and for certain $u \in X^{*}$ we have

$$
u(x) \leq u(z), \text { for all } x \in A,
$$

then $u(x) \leq u(z)$ is also a consequence of a finite subsystem of $\sigma$. The converse statement holds provided that $\sigma$ is linear. 
Proof. We only consider the non-trivial case $u \neq 0$.

(3.5) is equivalent to $u \in N_{A}(z) \backslash\{0\}$, and (3.3) entails the existence of

$$
v_{1} \in N_{C}(z) \text { and } v_{2} \in \text { cone }\left(\bigcup_{t \in T(z)} \partial f_{t}(z)\right)
$$

such that $u=v_{1}+v_{2}$.

If $v_{2}=0$, then $u=v_{1} \in N_{C}(z)$ and $u(x) \leq u(z)$ is a consequence of any possible subsystems of $\sigma$, whose solution set is always included in $C$.

If $v_{2} \neq 0$, the convexity of the subdifferential set entails the existence of $\lambda \in \mathbb{R}_{+}^{(T)}$ and $u_{t} \in \partial f_{t}(z), t \in$ $\operatorname{supp} \lambda \subset T(z)$, such that

$$
v_{2}=\sum_{t \in \operatorname{supp} \lambda} \lambda_{t} u_{t}
$$

Let

$$
\sigma_{1}:=\left\{f_{t}(x) \leq 0, t \in \operatorname{supp} \lambda ; x \in C\right\}
$$

and let $x \in A_{1}$, where $A_{1}$ is the solution set of $\sigma_{1}$. We have, for every $t \in \operatorname{supp} \lambda$,

$$
0 \geq f_{t}(x) \geq f_{t}(z)+u_{t}(x-z)=u_{t}(x-z)
$$

and so

$$
\begin{aligned}
0 & \geq \sum_{t \in \operatorname{supp} \lambda} \lambda_{t} f_{t}(x) \geq \sum_{t \in \operatorname{supp} \lambda} \lambda_{t} u_{t}(x-z) \\
& =v_{2}(x-z) \\
& =\left(u-v_{1}\right)(x-z) \\
& =u(x-z)+\left(-v_{1}\right)(x-z) \\
& \geq u(x-z)
\end{aligned}
$$

where the last inequality comes from $v_{1} \in N_{C}(z)$. Thus, we have proved that

$$
u(x) \leq u(z) \text {, for every } x \in A_{1} .
$$

Now let $C=X$ and $f_{t}(x)=a_{t}(x)-b_{t}$, with $a_{t} \in X^{*}$ and $b_{t} \in \mathbb{R}, t \in T$.

Let $u \in N_{A}(z) \backslash\{0\} ;$ i.e., $u(x-z) \leq 0$ for all $x \in A$. By assumption, there exists $S \subset T, S$ finite, such that $u(x) \leq u(z)$ if $a_{t}(x) \leq b_{t}$ for all $t \in S$.

By the same argument used in the proof of the converse in Theorem 1,

$$
(u, u(z)) \in K_{S}=\text { cone }\left\{\left(a_{t}, b_{t}\right), t \in S ;(0,1)\right\} .
$$

Then, there will exist $\lambda_{t} \geq 0, t \in S$, not all of them equal to zero, and $\mu \geq 0$ such that

$$
(u, u(z))=\sum_{t \in S} \lambda_{t}\left(a_{t}, b_{t}\right)+\mu(0,1),
$$

so that

$$
0=\sum_{t \in S} \lambda_{t}\left(a_{t}(z)-b_{t}\right)-\mu .
$$


Hence $\mu=0$ and $S \subset T(z)$, which entails

$$
u \in \text { cone }\left\{a_{t}, t \in T(z)\right\}=\text { cone }\left(\bigcup_{t \in T(z)} \partial f_{t}(z)\right)
$$

The second statement in the previous proposition does not hold for convex systems without any additional assumption, as the same Example 1 and any other finite convex system which is not LFM show. An example of infinite convex system (similar to [10], Ex. 2.1) that also illustrates this fact is the following.

Example 2. Let $X=C=\mathbb{R}, T=\mathbb{N}$, and

$$
\sigma=\left\{f_{t}(x):=\max \left\{0, x^{2 t+1}\right\} \leq 0, t \in T\right\}
$$

We observe that $A=]-\infty, 0]$, and for $z=0, T(0)=T$, and

$$
N_{A}(z)=\left[0,+\infty\left[\neq\{0\}=N_{C}(z)+\text { cone }\left(\bigcup_{t \in T(z)} \partial f_{t}(z)\right) .\right.\right.
$$

Thus, $\sigma$ is not LFM despite that the condition in the last proposition is satisfied (every finite subsystem has the same solution set).

\section{Extended FARKAS LEMMA}

From now on we use the following closedness condition [4] involving $f_{t}, t \in T, f$, and $C$ :

$$
\text { (CC) : The set epi } f^{*}+\operatorname{cl} K \text { is weak }{ }^{*} \text {-closed. }
$$

If epi $f^{*}+K$ is weak*-closed, then the closedness condition (CC) holds. The following theorem gives other sufficient conditions.

Theorem 1. If $\sigma$ is FM and $f$ is either linear or continuous at some point of $A$, then condition (CC) holds.

Proof. If $f$ is linear the statement is true by [9], Remark 5.6. So, we shall assume that $f$ is continuous at some point of $A$.

Let $h: X^{*} \rightarrow \mathbb{R} \cup\{+\infty\}$ be such that epi $h=K$.

Since we assume that $K$ is weak ${ }^{*}$-closed, then $h$ is a proper l.s.c. convex function, $g:=h^{*}$ satisfies the same properties, and

$$
\operatorname{epi} g^{*}=\operatorname{cone}\left\{\bigcup_{t \in T} \operatorname{epi} f_{t}^{*} \cup \operatorname{epi} \delta_{C}^{*}\right\} .
$$

Now we prove that $A \subset \operatorname{dom} g$. By assuming the contrary, let $x \in A$ such that $x \notin \operatorname{dom} g$.

Since $h^{*}(x)=+\infty$, there exists $v \in X^{*}$ such that $v(x)-h(v) \geq 1$. Then $(v, v(x)-1) \in$ epi $h$ and, by (2.1), we can write

$$
(v, v(x)-1)=\sum_{t \in S} \lambda_{t}\left(u_{t}, f^{*}\left(u_{t}\right)+\alpha_{t}\right)+\mu\left(w, \delta_{C}^{*}(w)+\beta\right),
$$

with $S \subset T,|S|<\infty,\left\{u_{t}, t \in S ; w\right\} \subset X^{*}$, and $\left\{\lambda_{t}, t \in S ; \alpha_{t}, t \in S ; \mu ; \beta\right\} \subset \mathbb{R}_{+}$.

From (4.1), we get

$$
1=\sum_{t \in S} \lambda_{t}\left(u_{t}(x)-f^{*}\left(u_{t}\right)-\alpha_{t}\right)+\mu\left(w(x)-\delta_{C}^{*}(w)-\beta\right) .
$$


On the other hand, since $x \in A$, we have

$$
u_{t}(x) \leq f_{t}(x)+f^{*}\left(u_{t}\right) \leq f^{*}\left(u_{t}\right), \forall t \in T,
$$

and, similarly,

$$
w(x) \leq \delta_{C}(x)+\delta_{C}^{*}(w) \leq \delta_{C}^{*}(w)
$$

Hence (4.2) yields $1 \leq 0$.

Finally, since $f$ is continuous at some point of $A \subset \operatorname{dom} g$, by Lemma 1, we conclude that epi $f^{*}+$ epi $g^{*}=$ epi $f^{*}+K$ is weak ${ }^{*}$-closed; i.e., (CC) holds.

If $\sigma$ is FM, then $f(x) \leq 0$ is consequence of $\sigma$ if and only if epif* $\subset K$ ([9], Cor. 4.2, extending [14], Th. 2.1). Since epi $(f-\alpha)^{*}=(0, \alpha)+$ epi $f^{*}$, we get that the convex inequality $f(x) \leq \alpha$ is consequence of $\sigma$ if and only if

$$
(0, \alpha)+\text { epif* } \subset K
$$

The next result provides a counterpart of (4.3) for the reverse-convex inequality $f(x) \geq \alpha$.

Lemma 4. Let $\sigma$ be FM and $\alpha \in \mathbb{R}$. Then $f(x) \geq \alpha$ is consequence of $\sigma$ if and only if

$$
(0,-\alpha) \in \operatorname{cl}(\text { epif* }+K)
$$

Proof. Assume that $f(x) \geq \alpha$ is consequence of $\sigma$. This means that $f(x) \geq \alpha$ for all $x \in A$, or equivalently, $\left(f+\delta_{A}\right)(x) \geq \alpha$ for all $x \in X$. Let $h(x)=\alpha$ for all $x \in X$. Then $f+\delta_{A} \geq h$ and it follows that $\left(f+\delta_{A}\right)^{*} \leq h^{*}$. This inequality, together with (2.2) and (3.1), implies that

$$
\left.(0,-\alpha) \in \operatorname{gph} h^{*} \subset \operatorname{epi}\left(f+\delta_{A}\right)^{*}=\operatorname{cl}\left\{\operatorname{epi} f^{*}+\operatorname{epi} \delta_{A}^{*}\right)\right\}=\operatorname{cl}\left\{\operatorname{epi} f^{*}+\operatorname{cl} K\right\} .
$$

Since

$$
\operatorname{cl}\left\{\text { epi } f^{*}+\operatorname{cl} K\right\}=\operatorname{cl}\left\{\text { epif } f^{*}+K\right\},
$$

(4.4) has been proved.

Now we assume that (4.4) holds. Let $\left\{\left(y^{\delta}, \beta^{\delta}\right), \delta \in \Delta\right\}$ be a net in

$$
\operatorname{epi} f^{*}+K=\operatorname{epi} f^{*}+\operatorname{epi} \delta_{C}^{*}+\operatorname{cone}\left\{\bigcup_{t \in T} \operatorname{epi} f_{t}^{*}\right\}
$$

converging to $(0,-\alpha)$. By $(2.1)$, for each $\delta \in \Delta$ there exist $z^{\delta} \in \operatorname{dom} f^{*}, \mu^{\delta} \geq 0, \lambda^{\delta} \in \mathbb{R}_{+}^{(T)}, u_{t}^{\delta} \in \operatorname{dom} f_{t}^{*}, \alpha_{t}^{\delta} \geq 0$ $\forall t \in T, v^{\delta} \in \operatorname{dom} \delta_{C}^{*}$, and $\gamma^{\delta} \geq 0$ such that

$$
\begin{aligned}
\left(y^{\delta}, \beta^{\delta}\right)= & \left(z^{\delta}, f^{*}\left(z^{\delta}\right)+\mu^{\delta}\right)+\left(v^{\delta}, \delta_{C}^{*}\left(v^{\delta}\right)+\gamma^{\delta}\right) \\
& +\sum_{t \in T} \lambda_{t}^{\delta}\left(u_{t}^{\delta}, f_{t}^{*}\left(u_{t}^{\delta}\right)+\alpha_{t}^{\delta}\right)
\end{aligned}
$$


Thus, given $x \in A$, we have

$$
\begin{aligned}
y^{\delta}(x)-\beta^{\delta}= & \left\langle\left(y^{\delta}, \beta^{\delta}\right),(x,-1)\right\rangle \\
= & z^{\delta}(x)-f^{*}\left(z^{\delta}\right)-\mu^{\delta}+v^{\delta}(x)-\delta_{C}^{*}\left(v^{\delta}\right)-\gamma^{\delta} \\
& +\sum_{t \in T} \lambda_{t}^{\delta}\left(u_{t}^{\delta}(x)-f_{t}^{*}\left(u_{t}^{\delta}\right)-\alpha_{t}^{\delta}\right) \\
\leq & f(x)-\mu^{\delta}+\delta_{C}(x)-\gamma^{\delta}+\sum_{t \in T} \lambda_{t}^{\delta}\left(f_{t}(x)-\alpha_{t}^{\delta}\right) \\
\leq & f(x)+\delta_{C}(x)+\sum_{t \in T} \lambda_{t}^{\delta} f_{t}(x) \leq f(x) .
\end{aligned}
$$

Hence $f(x) \geq \lim _{\delta}\left\{y^{\delta}(x)-\beta^{\delta}\right\}=\alpha$.

Semi-infinite versions of Lemma 4 , with $f_{t}: \mathbb{R}^{n} \rightarrow \mathbb{R}$ convex for all $t \in T$, are [16], Theorem 4.1 (where $C=\mathbb{R}^{n}$ and $f: \mathbb{R}^{n} \rightarrow \mathbb{R}$ ) and [3], Theorem 5.6. Observe that if $f$ is either linear or continuous at some point of $A$ then, by Theorem 1, we can replace (4.4) with

$$
(0,-\alpha) \in \operatorname{epi} f^{*}+K
$$

The previous result applies immediately to the set containment problem, which consists of deciding whether the solution set of a given system is contained in the solution set of another one. Dual characterizations of such set containments have played a key role in solving large scale knowledge-based data classification problems where they are used to describe the containments as inequality constraints in optimization problems. Recently, various extensions of the containment problem to general situations have been obtained in [23] and [16] by means of mathematical programming theory and conjugacy theory, respectively. One of the problems considered in [23] is the containment $A \subset B$, where $A$ is the solution set of $\sigma, C=X=\mathbb{R}^{n},|T|<\infty$, $B=\left\{x \in X \mid g_{s}(x) \geq 0, s \in S\right\},|S|<\infty$, all the involved functions being convex and differentiable (for obvious reasons, such a set $B$ is called reverse-convex). In [16], Theorem 4.1, all the involved functions are assumed to be finite-valued convex funtions on $\mathbb{R}^{n}$.

In the following extension $S$ and $T$ are arbitrary sets, and the functions $g_{s}: X \rightarrow \mathbb{R} \cup\{+\infty\}, s \in S$, are proper l.s.c. convex functions.

Corollary 1. Let $\sigma$ be FM. Then $A \subset B$ if and only if $0 \in \bigcap_{s \in S} \operatorname{cl}\left\{\operatorname{epi} g_{s}^{*}+K\right\}$.

Proof. $A \subset B$ if and only if $g_{s}(x) \geq 0$ is consequence of $\sigma$ for all $s \in S$. The conclusion follows from Lemma 4.

Now we give a new version of Farkas' lemma.

Theorem 2. If $\sigma$ is $\mathrm{FM},(\mathrm{CC})$ holds, and $\alpha \in \mathbb{R}$, then the following statements are equivalent to each other:

(i) $f(x) \geq \alpha$ is consequence of $\sigma$;

(ii) $(0,-\alpha) \in$ epif $^{*}+K$;

(iii) there exists $\lambda \in \mathbb{R}_{+}^{(T)}$ such that

$$
f(x)+\sum_{t \in T} \lambda_{t} f_{t}(x) \geq \alpha, \forall x \in C
$$

Proof. $[(i) \Rightarrow(i i)]$ It is a straightforward consequence of Lemma 4. 
$\left[(i i) \Rightarrow(\right.$ iii) $]$ Suppose that (ii) holds. Then, by (2.1), there exist $u \in \operatorname{dom} f^{*}, \beta \geq 0, u_{t} \in \operatorname{dom} f_{t}^{*}, r_{t} \geq 0$, $t \in T, v \in \operatorname{dom} \delta_{C}^{*}, r \geq 0$, and $\lambda \in \mathbb{R}_{+}^{(T)}$ such that

$$
(0,-\alpha)=\left(u, f^{*}(u)+\beta\right)+\sum_{t \in T} \lambda_{t}\left(u_{t}, f_{t}^{*}\left(u_{t}\right)+r_{t}\right)+\left(v, \delta_{C}^{*}(v)+r\right) .
$$

The last equality is equivalent to

$$
\left.\begin{array}{rl}
0 & =u+\sum_{t \in T} \lambda_{t} u_{t}+v \\
-\alpha & =f^{*}(u)+\beta+\sum_{t \in T} \lambda_{t}\left(f_{t}^{*}\left(u_{t}\right)+r_{t}\right)+\delta_{C}^{*}(v)+r
\end{array}\right\} .
$$

Since $f^{*}(u) \geq u(x)-f(x) \forall x \in X, f_{t}^{*}\left(u_{t}\right) \geq u_{t}(x)-f_{t}(x) \forall t \in T$ and $\forall x \in X$, and $\delta_{C}^{*}(v) \geq v(x) \forall x \in C$, it follows from (4.5) that, for all $x \in C$,

$$
\begin{aligned}
-\alpha & \geq u(x)-f(x)+\beta+\sum_{t \in T} \lambda_{t}\left(u_{t}(x)-f_{t}(x)+r_{t}\right)+v(x)+r \\
& \geq-f(x)-\sum_{t \in T} \lambda_{t} f_{t}(x)+\beta+\sum_{t \in T} \lambda_{t} r_{t}+r \\
& \geq-f(x)-\sum_{t \in T} \lambda_{t} f_{t}(x),
\end{aligned}
$$

which is (iii).

As the implication $[(i i i) \Rightarrow(i)]$ is obvious, the proof is complete.

Theorem 2 was established in [8], Theorem 2.2, under the assumption that $C=X$ is a Banach space, $\sigma$ is FM, and all the involved functions $\left(f, f_{t}, t \in T\right)$ are continuous. In the presence of a set constraint $C$, and assuming the continuity of the involved functions, the equivalence between (i) and (iii) was established in [12] under a closedness condition which is strictly stronger than the FM property [8]. By Theorem 1, the continuity of all the involved functions can be replaced by either the linearity of $f$ or by its continuity at some point in $A$.

\section{Optimality CONDitions}

In this section, and also in the next one, we consider the convex programming problem:

$$
\begin{aligned}
\text { Minimize } & f(x) \\
\text { subject to } & f_{t}(x) \leq 0, t \in T, \\
& x \in C,
\end{aligned}
$$

under the assumptions of Section 1.

In [4], Proposition 4.1, it is shown that, under condition (CC), $a \in A \cap \operatorname{dom} f$ is a minimizer of (P) if and only if $0 \in \partial f(a)+N_{A}(a)$. The next result provides a similar characterization involving the data (i.e., the constraint functions) instead of the feasible set $A$.

Theorem 3. Given the problem (P), assume that $\sigma$ is $\mathrm{FM}$, that $(\mathrm{CC})$ holds, and let $a \in A \cap \operatorname{dom} f$. Then $a$ is a minimizer of $(\mathrm{P})$ if and only if there exists $\lambda \in \mathbb{R}_{+}^{(T)}$ such that $\partial f_{t}(a) \neq \emptyset, \forall t \in \operatorname{supp} \lambda$, and the Karush-Kuhn-Tucker condition

$$
0 \in \partial f(a)+\sum_{t \in T} \lambda_{t} \partial f_{t}(a)+N_{C}(a) \text { and } \lambda_{t} f_{t}(a)=0, \forall t \in T
$$

holds.

Proof. The point $a \in A \cap \operatorname{dom} f$ is a minimizer of $(\mathrm{P})$ if and only if

$$
0 \in \partial\left(f+\delta_{A}\right)(a) .
$$


By the assumptions, epi $\delta_{A}^{*}=\operatorname{cl} K=K$ and epi $f^{*}+\operatorname{epi}_{A}^{*}$ is weak ${ }^{*}$-closed. Taking this fact into account, Lemma 2 ensures that (5.3) is equivalent to

$$
0 \in \partial f(a)+N_{A}(a)
$$

i.e., there exists $u \in \partial f(a)$ such that $u(x) \geq u(a)$ is consequence of $\sigma$.

First we assume that $a$ is a minimizer of $(\mathrm{P})$. Since $\sigma$ is FM, by Lemma 3 we have

$$
-(u, u(a)) \in K=\text { cone }\left\{\bigcup_{t \in T} \operatorname{epi} f_{t}^{*}\right\}+\operatorname{epi} \delta_{C}^{*}
$$

It follows from (2.1) and the representation (2.3), applied to $f_{t}^{*}$ and $\delta_{C}^{*}$, that there exist $\lambda \in \mathbb{R}_{+}^{(T)}, \varepsilon_{t} \geq 0, u_{t} \in$ $\partial_{\varepsilon_{t}} f_{t}(a) \forall t \in T, \gamma \geq 0, v \in \partial_{\gamma} \delta_{C}(a)$ satisfying

$$
-(u, u(a))=\left(\sum_{t \in T} \lambda_{t} u_{t}+v, \sum_{t \in T} \lambda_{t}\left[u_{t}(a)+\varepsilon_{t}-f_{t}(a)\right]+v(a)+\gamma-\delta_{C}(a)\right),
$$

which is equivalent to

$$
\left.\begin{array}{rl}
-u & =\sum_{t \in T} \lambda_{t} u_{t}+v \\
-u(a) & =\sum_{t \in T} \lambda_{t}\left[u_{t}(a)+\varepsilon_{t}-f_{t}(a)\right]+v(a)+\gamma-\delta_{C}(a)
\end{array}\right\}
$$

As $a \in C,(5.5)$ implies that

$$
\sum_{t \in T} \lambda_{t} \varepsilon_{t}-\sum_{t \in T} \lambda_{t} f_{t}(a)+\gamma=0
$$

Since $\lambda_{t} \varepsilon_{t} \geq 0,-\lambda_{t} f_{t}(a) \geq 0 \forall t \in T$, and $\gamma \geq 0$, we get $\lambda_{t} \varepsilon_{t}=0,-\lambda_{t} f_{t}(a)=0 \forall t \in T$, and $\gamma=0$. Thus, $\varepsilon_{t}=0$ whenever $t \in \operatorname{supp} \lambda$, and $-u(a)=\sum_{t \in T} \lambda_{t} u_{t}(a)+v(a)$, with $u_{t} \in \partial_{0} f_{t}(a)=\partial f_{t}(a) \forall t \in T$, and $v \in \partial_{0} \delta_{C}(a)=N_{C}(a)$. Therefore,

$$
0 \in \partial f(a)+\sum_{t \in T} \lambda_{t} \partial f_{t}(a)+N_{C}(a), \text { with } \lambda_{t} f_{t}(a)=0, \forall t \in T .
$$

The necessity is proved.

Conversely, if (5.2) holds for some $\lambda \in \mathbb{R}_{+}^{(T)}$ such that $\partial f_{t}(a) \neq \emptyset, \forall t \in \operatorname{supp} \lambda$, then there exists $u \in X^{*}$ such that $-u \in N_{C}(a)$ and

so that

$$
u \in \partial f(a)+\sum_{t \in T} \lambda_{t} \partial f_{t}(a) \subset \partial\left(f+\sum_{t \in T} \lambda_{t} f_{t}\right)(a)
$$

$$
f(x)+\sum_{t \in T} \lambda_{t} f_{t}(x) \geq f(a)+\sum_{t \in T} \lambda_{t} f_{t}(a)+u(x-a), \forall x \in X .
$$

Since $\lambda_{t} f_{t}(a)=0 \forall t \in T$, and $-u \in N_{C}(a)$, (5.6) implies

$$
f(x)+\sum_{t \in T} \lambda_{t} f_{t}(x)-f(a) \geq u(x-a) \geq 0, \quad \forall x \in C .
$$

Then, if $x \in A$

$$
f(x) \geq f(x)+\sum_{t \in T} \lambda_{t} f_{t}(x) \geq f(a),
$$

which proves $a$ to be a minimizer of $(\mathrm{P})$. 
It was shown in [9], Theorem 5.5, that (5.2) is a necessary and sufficient optimality condition for a point $a \in A \cap \operatorname{dom} f$ to be a minimizer of (P) assuming that $\sigma$ is FM, (CC) holds, and all the functions $f_{t}, t \in T$, are continuous at $a$. We have shown that the last assumption is superfluous.

Corollary 2. If the system $\sigma$ in (1.1) is FM, then it is also LFM.

Proof. If $z \in A$ and $u \in N_{A}(z) \backslash\{0\}$, the point $z$ turns out to be a minimum of the problem

$$
\begin{aligned}
\text { Minimize } & -u(x) \\
\text { subject to } & f_{t}(x) \leq 0, t \in T, \\
& x \in C
\end{aligned}
$$

Since $u$ is linear, Theorem 1 implies (CC), and Theorem 3 applies to conclude the existence of $\lambda \in \mathbb{R}_{+}^{(T)}$ such that

$$
0 \in\{-u\}+\sum_{t \in T} \lambda_{t} \partial f_{t}(z)+N_{C}(z)
$$

i.e.,

$$
u \in N_{C}(z)+\text { cone }\left(\bigcup_{t \in T(z)} \partial f_{t}(z)\right) .
$$

The converse of Corollary 2 fails even for linear semi-infinite systems (see, for instance, [11]).

The following theorem provides a counterpart in our context of the equivalence $(i) \Leftrightarrow($ iii $)$ in [21], Theorem 4.1 (we do not require the involved functions to be finite-valued).

Theorem 4. Let $a \in A$, the solution set of $\sigma$. The following statements are equivalent:

(i) $\sigma$ is LFM at a;

(ii) for any l.s.c. convex function $f$, with $a \in \operatorname{dom} f$, and such that $f$ is continuous at some point of $A$, the point $a$ is a minimizer of $f$ on $A$ if and only if there exists $\lambda \in \mathbb{R}_{+}^{(T)}$ such that $\partial f_{t}(a) \neq \emptyset, \forall t \in \operatorname{supp} \lambda$, and (5.2) is satisfied.

Proof. $[(i) \Rightarrow(i i)]$ The only thing that we have to prove is that if $a$ is a minimizer of $(\mathrm{P})$, then there exists $\lambda \in \mathbb{R}_{+}^{(T)}$ such that $\partial f_{t}(a) \neq \emptyset, \forall t \in \operatorname{supp} \lambda$, and (5.2) is satisfied.

Since $a \in A$ is a minimizer of $(\mathrm{P})$, thanks to Lemmas 1 and 2, and to the LFM property, we can write

$$
\begin{aligned}
0 & \in \partial\left(f+\delta_{A}\right)(a)=\partial f(a)+N_{A}(a) \\
& =\partial f(a)+\text { cone }\left(\bigcup_{t \in T(a)} \partial f_{t}(a)\right)+N_{C}(a),
\end{aligned}
$$

which brings the aimed conclusion.

$[(i i) \Rightarrow(i)]$ We have just to repeat the argument in the proof of Corollary 2.

\section{DuAlity AND STABility}

In this section we introduce a family of perturbed problems associated with the infinite convex optimization problem $(\mathrm{P})$ introduced in Section 1, but assuming now that all the involved functions, $f, f_{t} t \in T$, are finitevalued. We then consider the Lagrange dual problem of $(\mathrm{P})$, denoted by $(\mathrm{D})$. It is shown that, under the assumptions that $\sigma$ is FM and (CC) holds, we get strong duality between (P) and (D). Some kind of stability for $(\mathrm{P})$ is also analyzed. 


\subsection{Duality and saddle point theorem}

The following basic duality scheme is similar to the one developed in [25] (see, also, [2], Sect. 2.5, and [27], Sect. 2.6).

Consider the parametric problem $\left(\mathrm{P}_{u}\right), u \in \mathbb{R}^{T}$,

$$
\begin{array}{lll}
\left(\mathrm{P}_{u}\right) \quad \text { Minimize } & f(x) \\
\text { subject to } & f_{t}(x) \leq u_{t}, t \in T, \\
& x \in C,
\end{array}
$$

where $u:=\left(u_{t}\right) \in \mathbb{R}^{T}$, and whose feasible set is $A_{u}\left(A_{u}\right.$ can be empty for some $\left.u \neq 0\right)$. We represent by $h(u)$ the optimal value of $\left(\mathrm{P}_{u}\right)$. Then, $h(0)=\mathrm{v}(\mathrm{P})$.

If we define the function $\psi: X \times \mathbb{R}^{T} \rightarrow \mathbb{R} \cup\{+\infty\}$

$$
\psi(x, u):=f(x)+\delta_{A_{u}}(x),
$$

we can write

$$
\left(\mathrm{P}_{u}\right) \quad \text { Minimize } \psi(x, u), \quad x \in X,
$$

and obviously,

$$
(\mathrm{P}) \equiv\left(\mathrm{P}_{0}\right) \quad \text { Minimize } \quad \psi(x, 0), \quad x \in X .
$$

By a standard argument we see that $\psi$ is a proper l.s.c. convex function, whereas the optimal value function $h: \mathbb{R}^{T} \rightarrow \mathbb{R} \cup\{ \pm \infty\}$ is convex (possibly non-proper).

Now let $\psi^{*}$ be the conjugate of $\psi$ with respect to $(x, u) \in X \times \mathbb{R}^{T}$. Then, for each $\left(x^{*}, \eta\right) \in X^{*} \times \mathbb{R}^{(T)}$ we have

$$
\begin{aligned}
\psi^{*}\left(x^{*}, \eta\right) & =\sup _{x \in X, u \in \mathbb{R}^{T}}\left\{x^{*}(x)+\eta(u)-\psi(x, u)\right\} \\
& =\sup _{x \in X} \sup _{u \in \mathbb{R}^{T}}\left\{x^{*}(x)+\sum_{t \in T} \eta_{t} u_{t}-\delta_{A_{u}}(x)-f(x)\right\} \\
& = \begin{cases}\sup _{x \in C}\left\{x^{*}(x)+\sum_{t \in T} \eta_{t} f_{t}(x)-f(x)\right\}, & \text { if } \eta_{t} \leq 0, \forall t \in T, \\
+\infty, & \text { otherwise. }\end{cases}
\end{aligned}
$$

Thus,

$$
\psi^{*}(0, \lambda)= \begin{cases}-\inf _{x \in C}\left\{f(x)+\sum_{t \in T} \lambda_{t} f_{t}(x)\right\}, & \text { if } \lambda \in \mathbb{R}_{+}^{(T)} \\ +\infty, & \text { otherwise }\end{cases}
$$

On the other hand,

$$
\begin{aligned}
h^{*}(\lambda) & =\sup _{u \in \mathbb{R}^{T}}\left\{\lambda(u)-\inf _{x \in X} \psi(x, u)\right\} \\
& =\sup _{x \in X, u \in \mathbb{R}^{T}}\{\lambda(u)-\psi(x, u)\} \\
& =\psi^{*}(0, \lambda) .
\end{aligned}
$$

The dual problem of $(\mathrm{P})$ is defined as

$$
\text { (D) Maximize }-\psi^{*}(0, \lambda), \quad \lambda \in \mathbb{R}_{+}^{(T)} \text {. }
$$

Since the so-called Lagrangian function for $(\mathrm{P}), L: X \times \mathbb{R}^{(T)} \rightarrow \mathbb{R} \cup\{+\infty\}$, is

$$
L(x, \lambda):= \begin{cases}f(x)+\sum_{t \in T} \lambda_{t} f_{t}(x), & \text { if } x \in C \text { and } \lambda \in \mathbb{R}_{+}^{(T)}, \\ +\infty, & \text { otherwise, }\end{cases}
$$

it turns out that

$$
\text { (D) Maximize } \inf _{x \in C} L(x, \lambda), \quad \lambda \in \mathbb{R}_{+}^{(T)} \text {. }
$$


It follows from (6.1) that

$$
\begin{aligned}
\mathrm{v}(\mathrm{P}) & =h(0) \geq h^{* *}(0)=\sup _{\lambda \in \mathbb{R}_{+}^{(T)}}\left\{-h^{*}(\lambda)\right\} \\
& =\sup _{\lambda \in \mathbb{R}_{+}^{(T)}}\left\{-\psi^{*}(0, \lambda)\right\}=\mathrm{v}(\mathrm{D}),
\end{aligned}
$$

and the weak duality holds between $(\mathrm{P})$ and $(\mathrm{D})$. The next result shows that the strong duality holds between (P) and (D) under the assumptions that $\sigma$ is FM and that (CC) is satisfied for (P).

Theorem 5. If $(\mathrm{P})$ is bounded, $\sigma$ is FM, and $(\mathrm{CC})$ holds, then $v(\mathrm{D})=v(\mathrm{P})$ and $(\mathrm{D})$ is solvable.

Proof. Let $\alpha:=\mathrm{v}(\mathrm{P}) \in \mathbb{R}$. By definition of $\alpha$ we have

$$
f_{t}(x) \leq 0, t \in T, x \in C \Rightarrow f(x) \geq \alpha \text {. }
$$

Since $\sigma$ is FM and (CC) holds, it follows from Theorem 2 that there exists $\bar{\lambda} \in \mathbb{R}_{+}^{(T)}$ such that

$$
f(x)+\sum_{t \in T} \bar{\lambda}_{t} f_{t}(x) \geq \alpha, \forall x \in C,
$$

which implies

$$
\inf _{x \in C} L(x, \bar{\lambda}) \geq \alpha=\mathrm{v}(\mathrm{P}) .
$$

This, together with the weak duality, gives rise to

$$
\mathrm{v}(\mathrm{D})=\mathrm{v}(\mathrm{P})
$$

and $\bar{\lambda}$ is a maximizer of $(\mathrm{D})$.

Theorem 6. Suppose that $\sigma$ is FM and that (CC) holds. Then a point a $\in A$ is minimizer of $(\mathrm{P})$ if and only if there exists $\bar{\lambda} \in \mathbb{R}_{+}^{(T)}$ such that $(a, \bar{\lambda})$ is a saddle point of the Lagrangian function $L$, that is,

$$
L(a, \lambda) \leq L(a, \bar{\lambda}) \leq L(x, \bar{\lambda}), \quad \forall \lambda \in \mathbb{R}_{+}^{(T)} \text { and } \forall x \in C
$$

In this case, $\bar{\lambda}$ is a maximizer of $(\mathrm{D})$.

Proof. Let $a \in A$ be a minimizer of $(\mathrm{P})$. Then by an argument similar to the one in the proof of Theorem 5 , there exists $\bar{\lambda} \in \mathbb{R}_{+}^{(T)}$ such that

$$
f(x)+\sum_{t \in T} \bar{\lambda}_{t} f_{t}(x) \geq f(a), \forall x \in C .
$$

It follows from this inequality (by letting $x=a$ ) that $\sum_{t \in T} \bar{\lambda}_{t} f_{t}(a)=0$ and hence,

$$
L(x, \bar{\lambda}) \geq L(a, \bar{\lambda})=f(a), \forall x \in C .
$$

On the other hand, for each $\lambda \in \mathbb{R}_{+}^{(T)}$, and since $f_{t}(a) \leq 0$, we have

$$
L(a, \lambda)=f(a)+\sum_{t \in T} \lambda_{t} f_{t}(a) \leq f(a)=L(a, \bar{\lambda}) .
$$

Thus,

$$
L(a, \lambda) \leq L(a, \bar{\lambda}) \leq L(x, \bar{\lambda}), \forall \lambda \in \mathbb{R}_{+}^{(T)} \text { and } \forall x \in C,
$$


which proves that $(a, \bar{\lambda})$ is a saddle point of $L$.

Conversely, if there exists $\bar{\lambda} \in \mathbb{R}_{+}^{(T)}$ such that (6.4) holds then, by letting $\lambda=0$ in (6.4), we get

$$
f(a) \leq f(x)+\sum_{t \in T} \bar{\lambda}_{t} f_{t}(x), \forall x \in C .
$$

Thus, if $x \in A$ then $f(x) \geq f(a)$ as $\sum_{t \in T} \bar{\lambda}_{t} f_{t}(x) \leq 0$. This means that $a$ is a minimizer of $(\mathrm{P})$.

Finally, we have seen that (6.4) implies (6.5). Then

$$
\mathrm{v}(\mathrm{P})=f(a) \leq \inf _{x \in C} L(x, \bar{\lambda}) \leq \mathrm{v}(\mathrm{D}),
$$

and we conclude that $\bar{\lambda}$ is a maximizer of (D) by the weak duality.

\subsection{Stability}

We now recall two stability concepts for the problem (P) which were used in [20] (see also [7]).

Definition 3. (i) (P) is called inf-stable if $h(0)$ is finite and $h$ is 1.s.c. at 0 .

(ii) (P) is called inf-dif-stable if $h(0)$ is finite and there exists $\lambda_{0} \in \mathbb{R}^{(T)}$ such that

$$
h^{\prime}(0, u) \geq \lambda_{0}(u), \forall u \in \mathbb{R}^{T},
$$

where $h^{\prime}(0, u)$ is the directional derivative of $h$ at 0 in the direction $u$.

The proof of the following result is rather similar to the proof of [20], Theorem 7.3.2 (see also [27], Th. 2.6.1 (v)).

Lemma 5. The following properties are equivalent:

(i) (P) is inf-stable;

(ii) strong duality holds for $(\mathrm{P})$ and $(\mathrm{D})$ (i.e., $v(\mathrm{D})=v(\mathrm{P})$ ), and the values of these problems are finite.

The condition (ii) in Lemma 5 is called normality in [27]. Now we turn to the inf-dif-stability of (P). We begin by introducing a characterization of the inf-dif-stability, which proof is also quite similar to that given in [20], Proposition 7.3.7 and, so, it is also omitted.

Lemma 6. The problem $(\mathrm{P})$ is inf-dif-stable if and only if $\partial h(0) \neq \emptyset$.

In [2] it is asserted that the last condition is, in our convex setting, equivalent to the notion of calmness suggested in [6]. The relations between both notions of stability and normality are given in the following theorem.

Lemma 7. The following statements are equivalent:

(i) (P) is inf-dif-stable;

(ii) strong duality holds between $(\mathrm{P})$ and $(\mathrm{D})$, and $(\mathrm{D})$ is solvable;

(iii) (P) is inf-stable and $(\mathrm{D})$ is solvable.

Proof. Observe first that the equivalence between (ii) and (iii) follows from Lemma 5. Moreover, the equivalence between (i) and (ii) coincides, just taking into consideration Lemma 5, with [27], Theorem 2.6.1 (vi) (see also [2], Th. 2.142). Nevertheless, we give here an alternative constructive proof which is not based on the Young-Fenchel theorem.

$[(i) \Rightarrow(i i)]$ Suppose that $\left(\mathrm{P}_{u}\right)$ is inf-dif-stable. Then by Lemma $6, \partial h(0) \neq \emptyset$, and let $\tilde{\lambda} \in \mathbb{R}^{(T)}$ be a subgradient of $h$ at 0 . Then, for each $u \in \mathbb{R}^{T}$,

$$
h(u)-h(0) \geq \tilde{\lambda}(u)
$$


Let $\bar{\lambda}:=-\tilde{\lambda}$. We claim firstly that $\bar{\lambda} \in \mathbb{R}_{+}^{(T)}$. Assume, on the contrary, that $\bar{\lambda} \notin \mathbb{R}_{+}^{(T)}$. Note that $\mathbb{R}_{+}^{(T)}$ is a weak*-closed convex cone in $\mathbb{R}^{(T)}$. By the separation theorem, applied to the weak ${ }^{*}$-closed convex set $\mathbb{R}_{+}^{(T)}$ and the weak*-compact set $\{\bar{\lambda}\}$, there exists $\mu \in \mathbb{R}_{+}^{T}$ such that

$$
\bar{\lambda}(\mu)=-1 \text { and } z^{*}(\mu) \geq 0 \text { for all } z^{*} \in \mathbb{R}_{+}^{(T)} .
$$

Take $s>0$ arbitrarily. It follows from (6.6) that

$$
h(s \mu)-h(0) \geq \tilde{\lambda}(s \mu)=-\bar{\lambda}(s \mu)=-s \bar{\lambda}(\mu)=s>0 .
$$

On the other hand, since $\mathrm{v}(\mathrm{P})$ is finite, there exists a minimizing sequence $\left\{a_{n}\right\}_{n=1}^{\infty} \subset A$ of $(\mathrm{P})$, i.e., $\lim _{n \rightarrow \infty} f\left(a_{n}\right)=\mathrm{v}(\mathrm{P})$. Therefore, for any fixed $n \in \mathbb{N}, f_{t}\left(a_{n}\right) \leq 0$ for all $t \in T$. Since $\mu=\left(\mu_{t}\right) \in \mathbb{R}_{+}^{T}$ and $s>0, f_{t}\left(a_{n}\right) \leq s \mu_{t}$ for each $t \in T$, which means that $a_{n}$ is a feasible point for $\left(\mathrm{P}_{u}\right)$, with $u=s \mu$. Thus, $h(s \mu) \leq f\left(a_{n}\right)$. As the last inequality holds for arbitrary $n \in \mathbb{N}$, we get $h(s \mu) \leq \mathrm{v}(\mathrm{P})$ or, equivalently, $h(s \mu)-h(0) \leq 0$, which contradicts (6.7). Consequently, $\bar{\lambda} \in \mathbb{R}_{+}^{(T)}$.

Now, take $x \in C$. Then $f_{t}(x) \leq \bar{u}_{t}$ for all $t \in T$ where $\bar{u}=\left(\bar{u}_{t}\right) \in \mathbb{R}^{T}$ and $\bar{u}_{t}=f_{t}(x), t \in T$. This means that $x$ is a feasible point for $\left(\mathrm{P}_{\bar{u}}\right)$, and hence, $h(\bar{u}) \leq f(x)$. It follows from this and from (6.6) that

$$
\begin{aligned}
h(0) & \leq h(\bar{u})+\bar{\lambda}(\bar{u})=h(\bar{u})+\sum_{t \in T} \bar{\lambda}_{t} f_{t}(x) \\
& \leq f(x)+\sum_{t \in T} \bar{\lambda}_{t} f_{t}(x)=L(x, \bar{\lambda}) .
\end{aligned}
$$

Consequently, (6.8) holds for all $x \in C$ and hence,

$$
h(0)=\mathrm{v}(\mathrm{P}) \leq \inf _{x \in C} L(x, \bar{\lambda}) .
$$

It follows from this and from the weak duality that $\mathrm{v}(\mathrm{P})=\mathrm{v}(\mathrm{D})$ and that $\bar{\lambda} \in \mathbb{R}_{+}^{(T)}$ is an optimal solution of $(\mathrm{D})$.

$[(i i) \Rightarrow(i)]$ Suppose that $(i i)$ holds. Let $\bar{\lambda}$ be an optimal solution of (D). Then

$$
h(0)=\mathrm{v}(\mathrm{D})=\inf _{x \in C} L(x, \bar{\lambda}) \leq f(x)+\sum_{t \in T} \bar{\lambda}_{t} f_{t}(x), \forall x \in C .
$$

Let $u \in \mathbb{R}^{T}$ arbitrary. We consider first the case where the feasible set of $\left(\mathrm{P}_{u}\right)$ is non-empty. If $x \in C$ with $f_{t}(x) \leq u_{t}$ for all $t \in T$, then $\bar{\lambda}_{t}\left(f_{t}(x)-u_{t}\right) \leq 0$ for all $t \in T$. It then follows from (6.10) that, for each feasible point $x$ of $\left(\mathrm{P}_{u}\right)$,

or equivalently,

$$
f(x)+\sum_{t \in T} \bar{\lambda}_{t} f_{t}(x)-\sum_{t \in T} \bar{\lambda}_{t}\left(f_{t}(x)-u_{t}\right) \geq h(0)
$$

$$
f(x) \geq h(0)+(-\bar{\lambda})(u)
$$

Since the last inequality holds for each feasible point $x$ of $\left(\mathrm{P}_{u}\right)$, we get

$$
h(u) \geq h(0)+(-\bar{\lambda})(u) .
$$

If the feasible set of $\left(\mathrm{P}_{u}\right)$ is empty, then $h(u)=+\infty$ and (6.11) holds. Consequently, (6.11) holds for all $u \in \mathbb{R}^{T}$, which proves $-\bar{\lambda} \in \partial h(0)$. This and Lemma 6 together imply that $(\mathrm{P})$ is inf-dif-stable, and $(i)$ is proved. The proof is complete.

We are now in a position to give a sufficient condition for the inf-dif-stability of $(\mathrm{P})$. 
Theorem 7. If $(\mathrm{P})$ is bounded, $\sigma$ is $\mathrm{FM}$, and $(\mathrm{CC})$ holds, then $(\mathrm{P})$ is inf-dif-stable (and hence, inf-stable).

Proof. Under the assumptions of the theorem, it follows from Theorem 5 that the strong duality holds between (P) and (D), and the problem (D) is solvable. The conclusion of the theorem follows from Lemma 7.

Results of this type are also discussed and summarized in [26] for $(\mathrm{P})$, under the extra assumptions that $X$ is a Banach space, that all the involved functions are real-valued, that $T$ is a compact Hausdorff space, and that $G: X \rightarrow \mathbb{R}^{T}$, defined as $G(x)(t):=f_{t}(x)$, has continuous images (i.e., $\left.G(x) \in \mathcal{C}(T) \forall x \in X\right)$. Sufficient conditions for such a problem (P) to be inf-dif-stable (as in Th. 7) are also given in [26], assuming the continuity of $G: X \rightarrow \mathcal{C}(T)$ and a Slater-type c.q. (which is strictly stronger than the FM c.q. (see [19])). Sufficient conditions for the inf-stability in other context (non-convex objective function and linear equality constraints) are given in [1].

\section{REFERENCES}

[1] A. Auslender and M. Teboulle, Asymptotic Cones and Functions in Optimization and Variational Inequalities. Springer-Verlag, New York (2003).

[2] J.F. Bonnans and A. Shapiro, Perturbation Analysis of Optimization Problems. Springer-Verlag, New York (2000).

[3] R.I. Bot and G. Wanka, Farkas-type results with conjugate functions. SIAM J. Optim. 15 (2005) 540-554.

[4] R.S. Burachik and V. Jeyakumar, Dual condition for the convex subdifferential sum formula with applications. J. Convex Anal. 12 (2005) 279-290.

[5] A. Charnes, W.W. Cooper and K.O. Kortanek, On representations of semi-infinite programs which have no duality gaps. Manage. Sci. 12 (1965) 113-121.

[6] F.H. Clarke, A new approach to Lagrange multipliers. Math. Oper. Res. 2 (1976) 165-174.

[7] B.D. Craven, Mathematical Programming and Control Theory. Chapman and Hall, London (1978).

[8] N. Dinh, V. Jeyakumar and G.M. Lee, Sequential Lagrangian conditions for convex programs with applications to semidefinite programming. J. Optim. Theory Appl. 125 (2005) 85-112.

[9] N. Dinh, M.A. Goberna and M.A. López, From linear to convex systems: consistency, Farkas' lemma and applications. J. Convex Anal. 13 (2006) 279-290.

[10] M.D. Fajardo and M.A. López, Locally Farkas-Minkowski systems in convex semi-infinite programming. J. Optim. Theory Appl. 103 (1999) 313-335.

[11] M.A. Goberna and M.A. López, Linear Semi-infinite Optimization. J. Wiley, Chichester (1998).

[12] J. Gwinner, On results of Farkas type. Numer. Funct. Anal. Appl. 9 (1987) 471-520.

[13] J.-B. Hiriart Urruty and C. Lemarechal, Convex Analysis and Minimization Algorithms I. Springer-Verlag, Berlin (1993).

[14] V. Jeyakumar, Asymptotic dual conditions characterizing optimality for infinite convex programs. J. Optim. Theory Appl. 93 (1997) 153-165.

[15] V. Jeyakumar, Farkas' lemma: Generalizations, in Encyclopedia of Optimization II, C.A. Floudas and P. Pardalos Eds., Kluwer, Dordrecht (2001) 87-91.

[16] V. Jeyakumar, Characterizing set containments involving infinite convex constraints and reverse-convex constraints. SIAM J. Optim. 13 (2003) 947-959.

[17] V. Jeyakumar, A.M. Rubinov, B.M. Glover and Y. Ishizuka, Inequality systems and global optimization. J. Math. Anal. Appl. 202 (1996) 900-919.

[18] V. Jeyakumar, G.M. Lee and N. Dinh, New sequential Lagrange multiplier conditions characterizing optimality without constraint qualifications for convex programs. SIAM J. Optim. 14 (2003) 534-547.

[19] V. Jeyakumar, N. Dinh and G.M. Lee, A new closed cone constraint qualification for convex optimization, Applied Mathematics Research Report AMR04/8, UNSW, 2004. Unpublished manuscript. http://www.maths.unsw.edu.au/applied/ reports/amr08.html

[20] P.-J. Laurent, Approximation et optimization. Hermann, Paris (1972).

[21] C. Li and K.F. Ng, On constraint qualification for an infinite system of convex inequalities in a Banach space. SIAM J. Optim. 15 (2005) 488-512.

[22] W. Li, C. Nahak and I. Singer, Constraint qualification for semi-infinite systems of convex inequalities. SIAM J. Optim. 11 (2000) 31-52.

[23] O.L. Mangasarian, Set containment characterization. J. Global Optim. 24 (2002) 473-480.

[24] R. Puente and V.N. Vera de Serio, Locally Farkas-Minkowski linear semi-infinite systems. TOP 7 (1999) 103-121.

[25] R.T. Rockafellar, Conjugate Duality and Optimization, CBMS-NSF Regional Conference Series in Applied Mathematics 16, SIAM, Philadelphia (1974).

[26] A. Shapiro, First and second order optimality conditions and perturbation analysis of semi-infinite programming problems, in Semi-Infinite Programming, R. Reemtsen and J. Rückmann Eds., Kluwer, Dordrecht (1998) 103-133.

[27] C. Zălinescu, Convex analysis in general vector spaces. World Scientific Publishing Co., NJ (2002). 